\title{
Myocardial adaptation as assessed by speckle tracking echocardiography after isolated mitral valve surgery for primary mitral regurgitation
}

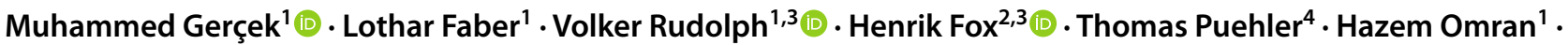 \\ Lisa Katharina Wolf ${ }^{1} \cdot$ Lech Paluszkiewicz ${ }^{2}$. Andreas M. Zeiher ${ }^{5} \cdot K_{\text {Kavous Hakim-Meibodi }}^{2}$ • Jan Gummert ${ }^{2,3}$. \\ Zisis Dimitriadis ${ }^{5}$
}

Received: 9 July 2020 / Accepted: 5 October 2020 / Published online: 13 October 2020

(c) The Author(s) 2020

\begin{abstract}
The risk of left ventricular (LV) and right ventricular (RV) maladaptation after surgery for isolated primary mitral regurgitation (PMR) is poorly defined. We aimed to evaluate LV and RV contractile function using speckle-tracking analysis alongside with quantification of exercise tolerance in patients with PMR after mitral valve surgery. All consecutive patients with symptomatic PMR undergoing mitral valve surgery between July 2015 and May 2017 were prospectively enrolled. Sequential echocardiographic studies along with clinical assessment were performed before and three months after surgery. Mean age in 138 patients was $65.8 \pm 12.7$ years, $48.2 \%$ (66) of whom were female. Mean LV ejection fraction decreased from $57 \pm 12 \%$ to $50 \pm 11 \%(\mathrm{p}<0.001), \mathrm{LV}$ global longitudinal strain deteriorated from $-19.2 \pm 4.1 \%$ to $-15.7 \pm 3.8 \%(\mathrm{p}<0.001)$ and mechanical strain dispersion increased from $88 \pm 12$ to $117 \pm 115 \mathrm{~ms}(\mathrm{p}=0.004)$. There was a reduction in tricuspid annulus plane systolic excursion from $22 \pm 5 \mathrm{~mm}$ to $18 \pm 4 \mathrm{~mm}(\mathrm{p}<0.001)$, as well as a slight deterioration of RV free wall mean longitudinal strain from $-16.9 \pm 5.6 \%$ to $-15.7 \pm 4.1 \%(\mathrm{p}=0.05)$. The rate of moderate to severe tricuspid regurgitation significantly decreased $(\mathrm{p}<0.005)$. Regarding exercise tolerance, the New York Heart Association class improved $(\mathrm{p}<0.001)$ and the walking distance increased $(\mathrm{p}<0.001)$. During mid-term follow up after surgery for PMR, a deterioration of LV and RV contractile function measures could be observed. However, the clinical status, LV dimensions, and concomitant tricuspid regurgitation improved which in particular imply more effective RV contractile pattern.
\end{abstract}

Keywords Mitral valve surgery $\cdot$ Strain analysis $\cdot$ Myocardial adaptation

Muhammed Gerçek

mugercek@hdz-nrw.de

1 Clinic for General and Interventional Cardiology/Angiology, Herz- Und Diabeteszentrum NRW, Ruhr-Universität Bochum, Georgstraße 11, 32545 Bad Oeynhausen, Germany

2 Clinic for Thoracic and Cardiovascular Surgery, HerzUnd Diabeteszentrum NRW, Ruhr-Universität Bochum, Bad Oeynhausen, Germany

3 Heart Failure Department, Herz- Und Diabeteszentrum NRW, Ruhr-Universität Bochum, Bad Oeynhausen, Germany

4 Department of Cardiac and Vascular Surgery, University Medical Center Schleswig Holstein, Campus Kiel, Kiel, Germany

5 Department of Cardiology, University Hospital Frankfurt, Frankfurt am Main, Germany

\section{Introduction}

Primary mitral regurgitation (PMR) due to mitral valve degeneration is the most common etiology in patients undergoing mitral valve surgery [1]. Surgical mitral valve repair or replacement, if repair is unfeasible, is the treatment of choice in case of symptomatic severe PMR [2].

Yet, patients with mitral regurgitation are often referred too late for surgery due to alleged preserved left ventricular (LV) function in echocardiographic controls [3]. Due to the load dependence of standard echocardiographic parameters which are used for the assessment of LV function, LV ejection fraction may substantially overestimate myocardial performance $[4,5]$.

However, the risk of functional LV maladaptation, the reaction of right ventricular (RV) function, and the resulting clinical implications after mitral valve surgery for isolated 
mitral regurgitation are poorly defined [6]. On the other side, evaluation of RV function, particularly after cardiac surgery, is challenging due to the complexity of RV geometry, the high RV sensitivity to hemodynamical changes and ventricular interdependence [7].

Speckle-tracking based myocardial deformation analysis has meanwhile become an established method to evaluate myocardial function. Speckle-tracking based assessment of longitudinal strain is independent of the insonation angle, and can be used retrospectively on digitally archived standard grey-scale images [8].

Hence, we aimed to evaluate LV and RV contractile function using longitudinal strain by speckle-tracking analysis together with the clinical status of patients with isolated PMR before and 3 months after mitral valve surgery.

\section{Methods}

Assessment of exercise tolerance by the New York Heart Association (NYHA) classification alongside, the 6-min walking test and echocardiographic examinations were prospectively performed before and 3 months after surgery in all consecutive patients with severe PMR who underwent isolated mitral valve surgery between July 2015 and May 2017. The decision for surgical treatment was made after heart team discussion for each case individually. The study was approved by local Ethics Committee of Ruhr University of Bochum and carried out in accordance with the Declaration of Helsinki. All data were included in a database, which is registered at www.clinicaltrials.gov (NCT02296710).

\section{Standard echocardiography}

All study participants underwent standard transthoracic echocardiography (EPIQ seven, Philips Electronics, Netherlands). The echo studies were performed by highly qualified medical staff and analysed by the same echocardiographer with long-time experience. The analyses and grading of the mitral regurgitation were performed according to the recommendations of the American and European Societies of Echocardiography $[9,10]$.

In cases with irregular cardiac rhythm (e.g. atrial fibrillation, frequent atrial or ventricular ectopy) at least five loops were recorded and the average values has been provided.

LV ejection fraction was assessed using the Simpson's method. LV stroke volume was calculated by subtraction of the LV end-systolic volume from the end-diastolic volume. The Nyquist-limit was placed around $50-60 \mathrm{~cm} / \mathrm{s}$ in color Doppler settings.

To characterize RV function, tricuspid annular plane systolic excursion (TAPSE) and RV fractional area change
(RV-FAC) were measured alongside with RV free wall longitudinal strain analysis.

\section{Strain analyses}

LV global longitudinal strain (GLS) was assessed as previously described using the speckle-tracking algorithm provided within the QLAB system (QLAB Version 10.2) [11]. Through three apical views (four-chamber view, threechamber view, two-chamber view) the end-diastolic frame was selected and the endocardial contour was tracked manually (Fig. 1a-c).

RV free wall longitudinal strain assessment was performed using a RV focused view with optimized RV endocardial borders according to the recommendations of the European and American Societies of Echocardiography (Fig. 1d) [12]. The other frames of the cineloop were tracked automatically and adjusted manually, if needed. Additionally, strain dispersion was documented for each LV segment. Mechanical strain dispersion was calculated as the difference between the highest and the lowest value from time to peak strain assessed through the three apical planes [13].

\section{Statistical analysis}

Statistical analysis was performed using the SPSS-Software (Version 21, IBM Corporation, Armonk, NY, USA). Continuous variables are reported as mean \pm standard deviation. Categorical variables are presented as frequencies and percentages. Baseline data were validated for normal distribution using the Kolmogorov-Smirnov method. Student's T-test for unpaired and paired parametric samples or their analogues for nonparametric samples (Mann-Whitney and Wilcoxon signed rank) or the chi-square test were performed for group comparisons. A p-value $<0.05$ was considered significant for all comparisons.

\section{Results}

A total of 156 consecutive patients with primary mitral regurgitation were admitted and evaluated for mitral valve repair between July 2015 and May 2017. Five of them also required myocardial revascularization and four patients presented with a combined valve disease which had to be addressed. eight patients refused participation in the study and one patient was found to suffer from mitral valve endocarditis. Finally, 138 patients were included in the analyses. The baseline characteristics including parameters for mitral regurgitation severity are shown in Tables 1 and 2 . Patients' mean age was $65.8 \pm 12.7$ years, and $66(47.8 \%)$ of them were female. Mean EuroScore II was $2.6 \pm 2.8 \%$, defining a low to intermediate perioperative risk. Mean 


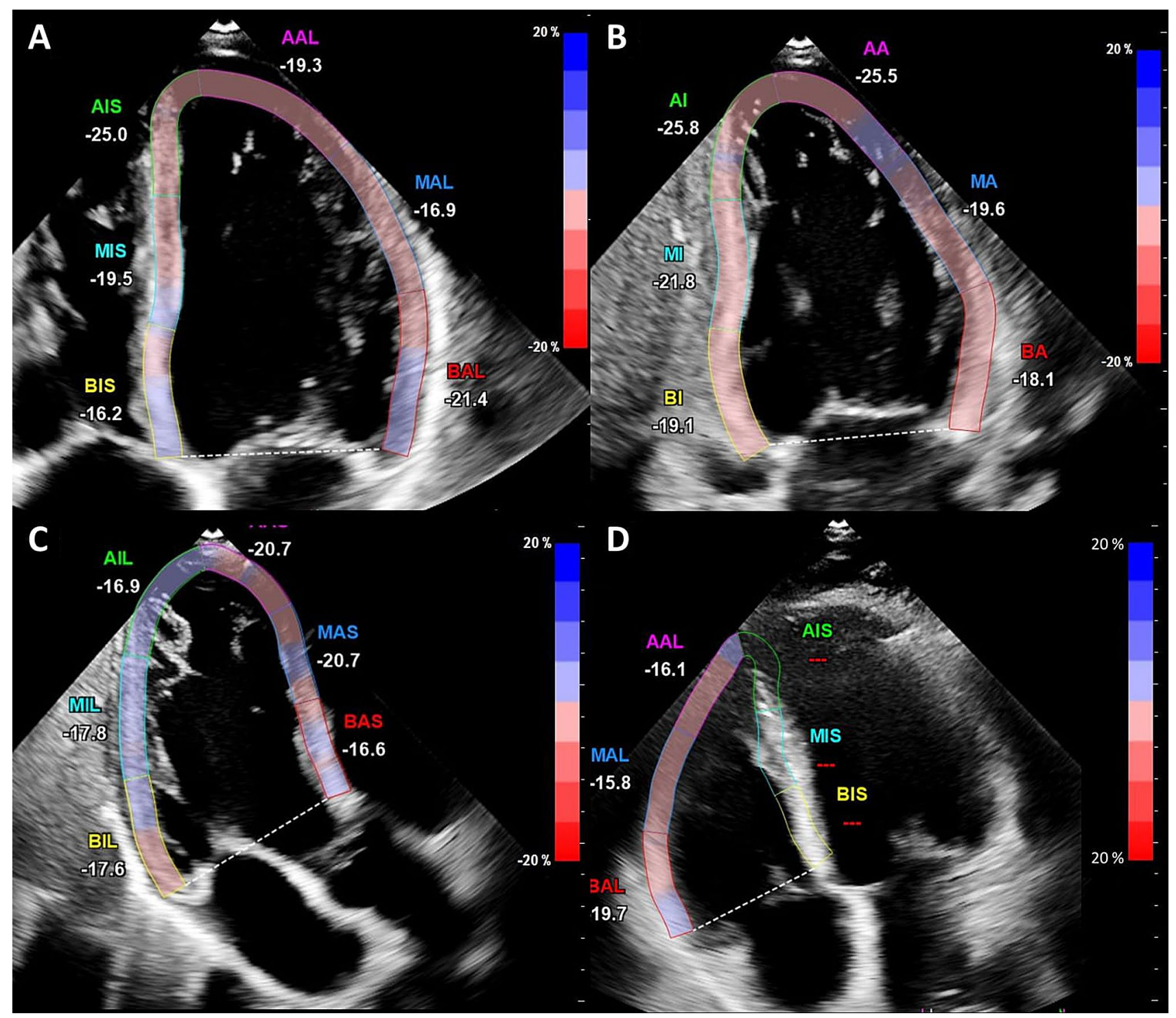

Fig. 1 Strain analysis of the left and right ventricle Apical four a two b three chamber view $\mathbf{c}$ and a right ventricular focus view $\mathbf{d}$ were used for strain analysis. An end-diastolic frame was selected for the left ventricle (with the interventricular septum) and for the right ventricular free wall (without the interventricular septum), and the

LV ejection fraction was $57 \pm 12 \%$, and degree of mitral regurgitation was characterized by an effective regurgitant volume of $43 \pm 3 \mathrm{~mm}^{2}$, a regurgitant volume of $67 \pm 7 \mathrm{ml}$ and a mean biplane vena contracta of $7.3 \pm 0.5 \mathrm{~mm}$. Out of the entire group 95 patients $(68.9 \%)$ underwent mitral valve repair and $43(31.1 \%)$ valve replacement.

Details of echocardiographic parameters before and after surgery are presented in Table 3 (left ventricle) and Table 4 (right ventricle). Three months after surgery, 121 patients $(87.7 \%$ ) had no residual MR and in 17 patients (12.3\%) only trivial MR was detectable. endocardial contour was manually tracked. The other frames were automatically tracked and corrected, if necessary. After verification the longitudinal strain was automatically calculated on average and regionally

LV end-diastolic volume markedly decreased from $157 \pm 57 \mathrm{ml}$ to $138 \pm 51 \mathrm{ml}(\mathrm{p}<0.001)$ following valve surgery, while the other morphological parameters such as end-systolic diameter, septal thickness and posterior wall thickness did not change.

Compared to baseline examinations, LV ejection fraction decreased from $57 \pm 12 \%$ to $50 \pm 11 \%(\mathrm{p}<0.001)$ while LV GLS deteriorated from $-19.2 \pm 4.1 \%$ to $-15.7 \pm 3.8 \%(\mathrm{p}<0.001)$. Additionally, strain dispersion increased from $88 \pm 12 \mathrm{~ms}$ to $117 \pm 115 \mathrm{~ms}(\mathrm{p}=0.004)$. 
Table 1 Collective-wide baseline characteristics

Baseline characteristics $(n=138)$

Age

Female

Coronary artery disease

Body mass index $\left[\mathrm{kg} / \mathrm{m}^{2}\right]$

EuroScore I [\%]

EuroScore II [\%]

Peripheral artery disease

Stroke

Diabetes mellitus

Renal insufficiency

Chronic obstructive pulmonary disease

Left bundle branch block

History of myocardial infarction

History of percutaneous coronary intervention

History of cardiac surgery

Atrial fibrillation

Pacemaker

$65.8 \pm 12.73(68)$
$47.8 \%(66)$
$10.9 \%(15)$
$26.4 \pm 4.3$
$8.6 \pm 8.5 \%$
$2.6 \pm 2.8 \%$
$4.3 \%(6)$
$11.6 \%(16)$
$8.7 \%(12)$
$10.1 \%(14)$
$7.2 \%(10)$
$2.2 \%(3)$
$4.3 \%(6)$
$5.1 \%(7)$
$11.6 \%(16)$
$34.8 \%(48)$
$3.6 \%(5)$

Table 2 Baseline echocardiographic parameters of the whole collective

\begin{tabular}{|c|c|}
\hline \multicolumn{2}{|l|}{ Baseline echocardiographic parameters } \\
\hline \multicolumn{2}{|l|}{$\begin{array}{l}\text { MV PISA radius adjusted to Nyquist limit } 30-40 \mathrm{~cm} / \mathrm{s} \\
{[\mathrm{mm}]}\end{array}$} \\
\hline MR vena contracta $[\mathrm{mm}]$ & $7.3 \pm 0.5$ \\
\hline MR effective regurgitant orifice area $\left[\mathrm{mm}^{2}\right]$ & $43 \pm 3$ \\
\hline MR regurgitant volume $[\mathrm{ml}]$ & $67 \pm 7$ \\
\hline LA maximal diameter length $[\mathrm{mm}]$ & $66 \pm 12$ \\
\hline LA maximal diameter width $[\mathrm{mm}]$ & $55 \pm 1$ \\
\hline LA volume $[\mathrm{ml}]$ & $137 \pm 74$ \\
\hline LA volume index $\left[\mathrm{ml} / \mathrm{m}^{2}\right]$ & $72 \pm 30$ \\
\hline LV EF Simpson [\%] & $57 \pm 12$ \\
\hline
\end{tabular}

$M V$ mitral valve, $M R$ mitral regurgitation, $L A$ left atrium, $L V$ left ventricle, $E F$ ejection fraction, PISA proximal isovelocity surface area

With respect to RV function, tricuspid annular plane systolic excursion (TAPSE) was reduced from $22 \pm 5 \mathrm{~mm}$ to $18 \pm 4 \mathrm{~mm}(\mathrm{p}<0.001)$. RV free wall mean strain also showed a slight although borderline significant deterioration (from $-16.9 \pm 5.6 \%$ to $-15.7 \pm 4.1 \% ; \mathrm{p}=0.05$ ) However, tricuspid regurgitation improved after mitral valve surgery (Fig. 2b). While $20.4 \%$ of the patients had a moderate to severe TR before mitral valve surgery, this figure decreased to $5.3 \% 3$ months after surgery with consecutively decreasing RA diameter (Table 4).

Regarding exercise tolerance, NYHA classification (at baseline $66.3 \%$ were in NYHA class III or IV, 3 months after surgery $85.2 \%$ were in NYHA class I or II; $p<0.001$ ) and walking distance in the 6-min walking test $(372 \pm 32 \mathrm{~m}$ to $425 \pm 117 \mathrm{~m} ; \mathrm{p}<0.001$ ) improved significantly (Fig. 2a, c).

\section{Discussion}

Due to the poorly defined risk for ventricular dysfunction after mitral surgery and its clinical impact, we evaluated the adaptation of the left and right ventricle after surgical mitral valve treatment in patients with severe mitral regurgitation and the clinical status before and 3 months after surgery.

\section{Left ventricular dysfunction after mitral valve surgery}

Mean LV GLS in our patients was $-19.2 \%$ at baseline and showed a deterioration after mitral valve surgery as an indicator for LV dysfunction. This is in accordance with the retrospective observation of Witkowski et al. who described a GLS worse than $-19.9 \%$ as an independent predictor for LV dysfunction in severe primary mitral regurgitation [14].

Hiemsatra et al. described LV GLS as independently associated with all-cause mortality and cardiovascular events in a cohort of 593 patient who underwent mitral valve surgery with a median follow-up of 6.4 years, (Hazard ratio 1.13; $95 \%$ confidence interval: 1.06 to $1.21 \mathrm{p}<0.001)$. In this study, LV-EF and LV GLS showed a similar deterioration of the contractile function (3). In a retrospectively analysed cohort of 506 patients with a wide range of cardiac comorbidities and a median follow-up of 3.5 years, Kim et al. postulated GLS to better predict cardiac events and allcause mortality than standard echocardiographic parameters (Multivariate Cox models HR 1.229 95\% CI: 1.135 to 1.331; $\mathrm{p}<0.001)$. The authors concluded this measure to be helpful to estimate the optimal timing for mitral valve surgery [15]. Interestingly, mechanical strain dispersion also increases after mitral valve surgery (Table 4). Prolonged mechanical strain dispersion is a sign for heterogeneity of systolic myocardial contraction due to the development of fibrosis formation and is associated with cardiac arrhythmias [16]. Therefore, strain dispersion could provide important information about cardiac remodeling during patient evaluation for mitral valve surgery [17].

However, despite functional impairment of the left ventricle, the patients showed pronounced clinical improvement in NYHA class and 6-min walking distance (Fig. 2a, c). Moreover, LA and LV diameter and volumes decreased after mitral valve surgery demonstrating a relevant reverse remodelling. By eliminating the regurgitation fraction of overall stroke volume, LV enlargement receded allowing for normal stress shortening $[18,19]$. However, since stroke volume and ejection fraction are required for antegrade flow only, myocardial performance is optimized and economized [20] whereas, 
Table 3 Morphological and functional changes of the left ventricle
Table 4 Morphologic and functional changes of the right ventricle

\begin{tabular}{lllc}
\hline Echocardiographic parameters & Before surgery & 3 months after surgery & p-value \\
\hline LA maximal diameter length [mm] & $66 \pm 11$ & $55 \pm 10$ & $<0.001$ \\
LA maximal diameter width [mm] & $55 \pm 9$ & $50 \pm 8$ & $<0.001$ \\
LA volume [ml] & $137 \pm 74$ & $97 \pm 51$ & $<0.001$ \\
LA volume index [ml/m $]$ & $72 \pm 30$ & $47 \pm 25$ & $<0.001$ \\
LVEDD [mm] & $58 \pm 7$ & $55 \pm 8$ & $<0.001$ \\
LVESD [mm] & $4.0 \pm 7$ & $4 \pm 9$ & 0.5 \\
Septum thickness [mm] & $9 \pm 2$ & $8 \pm 4$ & 0.4 \\
Post wall thickness [mm] & $9 \pm 2$ & $9 \pm 1$ & 0.6 \\
FS [\%] & $31 \pm 9$ & $28 \pm 11$ & 0.003 \\
LV end-diastolic volume [ml] & $157 \pm 57$ & $138 \pm 51$ & $<0.001$ \\
LV end-systolic volume [ml] & $67 \pm 31$ & $70 \pm 35$ & 0.2 \\
LV EF Simpson [\%] & $57 \pm 12$ & $50 \pm 11$ & $<0.001$ \\
Stroke volume [ml] & $93 \pm 39$ & $68 \pm 25$ & $<0.001$ \\
GLS [\%] & $-19.2 \pm 4.1$ & $-15.7 \pm 3.8$ & $<0.001$ \\
Mechanical strain dispersion [msec] & $88 \pm 12$ & $117 \pm 115$ & 0.004 \\
\hline
\end{tabular}

$L A$ left atrial, $L V$ left ventricular, $L V E D D$ left ventricular end-diastolic diameter, $L V E S D$ left ventricular end-systolic diameter, $F S$ fractional shortening, $G L S$ global longitudinal strain, $E F$ ejection fraction

\begin{tabular}{lllc}
\hline Echocardiographic parameters & Before surgery & 3 months after surgery & p-value \\
\hline RA maximal diameter length [mm] & $57 \pm 11$ & $54 \pm 10$ & 0.005 \\
RA maximal diameter width [mm] & $45 \pm 10$ & $44 \pm 8$ & 0.9 \\
RA volume [ml] & $78 \pm 54$ & $74 \pm 45$ & 0.8 \\
RV maximal diameter [mm] & $43 \pm 9$ & $43 \pm 8$ & 0.9 \\
TAPSE [mm] & $22 \pm 5$ & $18 \pm 4$ & $<0.001$ \\
RV end-diastolic area [mm2] & $19 \pm 6$ & $19 \pm 7$ & 0.4 \\
RV end-systolic area [mm2] & $11 \pm 5$ & $11 \pm 4$ & 0.2 \\
FAC [\%] & $42 \pm 12$ & $42 \pm 11$ & 0.7 \\
RV basal segment strain [\%] & $-15.6 \pm 5.5$ & $-14.9 \pm 4.5$ & 0.3 \\
RV middle segment strain [\%] & $-18.3 \pm 7.0$ & $-16.2 \pm 4.7$ & 0.004 \\
RV apical segment strain [\%] & $-17.1 \pm 6.8$ & $-15.9 \pm 5.0$ & 0.4 \\
RV mean strain [\%] & $-16.9 \pm 5.6$ & $-15.7 \pm 4.1$ & 0.05 \\
Tricuspid regurgitation degree [I-III] & $33.6 \% 0$ & $44.3 \% 0$ & $<0.001$ \\
& $46.0 \%$ I & $50.4 \%$ I & \\
& $15.1 \%$ II & $4.4 \%$ II & $0.9 \%$ III \\
TV PISA radius adjusted to Nyquist limit & $3.3 \% \pm$ III & $2.8 \pm 4.0$ & 0.04 \\
30-40 cm/s [mm] & & & 0.03 \\
TR vena contracta [mm] & $1.9 \pm 0.3$ & $1.1 \pm 0.3$ &
\end{tabular}

$F A C$ fractional area change, $R A$ right atrial, $R V$ right ventricular, TAPSE tricuspid annular plane systolic excursion, $T R$ tricuspid regurgitation, $T V$ tricuspid valve, $P I S A$ proximal isovelocity surface area 


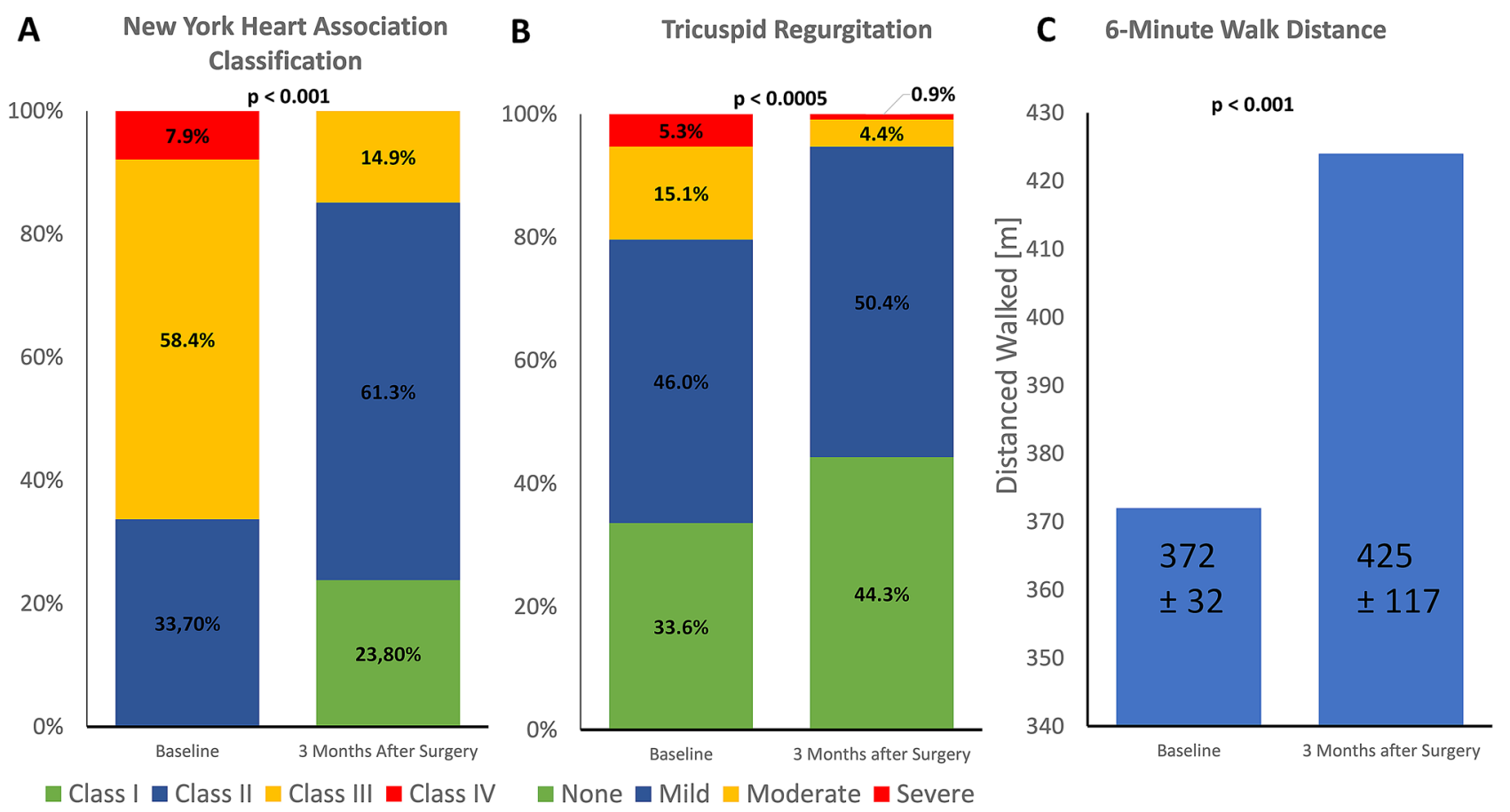

Fig. 2 Clinical Improvement and Reduction of Tricuspid Regurgitation after Mitral Valve Surgery Patients with symptomatic primary mitral regurgitation presented a noticeably clinical improvement accompanying the reduction of tricuspid regurgitation as a sign for economized right ventricular function. The New York Heart Associa-

according to our results, at least a temporary postoperative medical therapy to support myocardial unloading and reverse remodelling is suggested.

\section{Right ventricular dysfunction after mitral valve surgery}

Mitral regurgitation leads to a volume overload of the LA [18]. The LA is initially able to keep the pressure stable through its enlargement, but over time the pressure in the pulmonary venous system increases which eventually leads to an increased pulmonary artery pressure [7].

In the absence of volume overload after surgery, the pressure in the pulmonary vascular bed and consecutively in the right ventricle decreases. Right ventricular dimensions and functional tricuspid regurgitation are consecutively reduced [7]. However, as on the left side, some measures of RV function decreased. While FAC did not change, RV free wall strain and TAPSE were reduced. This deterioration is probably explained by geometric changes of the RV due to pericardial incision and the loss of pericardial support [21]. Depending on the pericardial incision and the surgical access path, parameters for the longitudinal RV function can show a decrease, despite overall normal global right ventricular function [21]. tion class improved significantly. At baseline $66.3 \%$ were in NYHA class III or IV. 3 months after surgery $85.2 \%$ were in NYHA class I or II $(\mathrm{p}<0.001)$ a The rate of moderate to severe tricuspid regurgitation decreases from $20.4 \%$ to $5.3 \%$ b The walking distance in the 6-min walking test increased from $372 \pm 32 \mathrm{~m}$ to $425 \pm 117 \mathrm{~m}(\mathrm{p}<0.001)(\mathbf{c})$

Another aspect is the reduced mobility of the septum due to the increased LV impairment. In addition, the incompletely understood cardioplegia effect may have played a role [3, 22-24]. The septal wall is involved in the mechanism of "squeezing out" the right ventricle. Together with the apex, the septal wall serves as an abutment to counteract the tension of the bellow-type right ventricle, and thus transports the blood towards the pulmonary arteries. About $24 \%$ of the RV function is taken over by the septal wall [7].

Our mid-term follow-up data on exercise tolerance demonstrate a clear clinical improvement, which implies an economization and higher effectiveness of RV myocardial performance. Accordingly, tricuspid regurgitation also improved after surgery probably because of improved hemodynamic and lack of volume overload which is also a sign of recovered clinical status $[25,26]$.

\section{Limitations}

The study is descriptive and not designed to explain the phenomena it observes and can therefore only generate hypotheses. In addition, further studies should investigate whether and to what extent the deteriorated function parameters 
persist during longer-term follow-up and whether this has a long-term impact on survival.

\section{Conclusion}

During mid-term follow up after surgery for PMR, a deterioration of $\mathrm{LV}$ and $\mathrm{RV}$ contractile function measures could be observed. However, the clinical status, LV dimensions, and concomitant tricuspid regurgitation improved significantly which in particular imply more effective RV contractile pattern.

Author contributions All authors have made substantial contributions to the manuscript, are responsible for the contents, and have read and approved the manuscript for submission to the International Journal of Cardiovascular Imaging.

Funding Open Access funding enabled and organized by Projekt DEAL. The study was supported by the Medical Faculty, Ruhr-Universität Bochum, Germany (FoRUM programme F811-14).

Data Availability All presented data are available and will be issued if necessary.

\section{Compliance with ethical standards}

\section{Conflict of interest None.}

Open Access This article is licensed under a Creative Commons Attribution 4.0 International License, which permits use, sharing, adaptation, distribution and reproduction in any medium or format, as long as you give appropriate credit to the original author(s) and the source, provide a link to the Creative Commons licence, and indicate if changes were made. The images or other third party material in this article are included in the article's Creative Commons licence, unless indicated otherwise in a credit line to the material. If material is not included in the article's Creative Commons licence and your intended use is not permitted by statutory regulation or exceeds the permitted use, you will need to obtain permission directly from the copyright holder. To view a copy of this licence, visit http://creativecommons.org/licenses/by/4.0/.

\section{References}

1. Fonarow GC, Yancy CW, Hernandez AF, Peterson ED, Spertus JA, Heidenreich PA (2011) Potential impact of optimal implementation of evidence-based heart failure therapies on mortality. Am Heart J 161:1024-30.e3

2. Baumgartner H, Falk V, Bax JJ, De Bonis M, Hamm C, Holm PJ et al (2017) 2017 ESC/EACTS guidelines for the management of valvular heart disease. Eur Heart J 38:2739-2791

3. Hiemstra YL, Tomsic A, van Wijngaarden SE, Palmen M, Klautz RJM, Bax JJ et al (2020) Prognostic value of global longitudinal strain and etiology after surgery for primary mitral regurgitation. JACC Cardiovasc Imag 13:577-585

4. Lee R, Haluska B, Leung DY, Case C, Mundy J, Marwick TH (2005) Functional and prognostic implications of left ventricular contractile reserve in patients with asymptomatic severe mitral regurgitation. Heart 91:1407-1412

5. Starling MR, Kirsh MM, Montgomery DG, Gross MD (1993) Impaired left ventricular contractile function in patients with longterm mitral regurgitation and normal ejection fraction. J Am Coll Cardiol 22:239-250

6. Zoghbi WA, Adams D, Bonow RO, Enriquez-Sarano M, Foster E, Grayburn PA et al (2017) Recommendations for noninvasive evaluation of native valvular regurgitation: a report from the american society of echocardiography developed in collaboration with the society for cardiovascular magnetic resonance. J Am Soc Echocardiogr 30:303-371

7. Del Rio JM, Grecu L, Nicoara A (2019) Right ventricular function in left heart disease. Semin Cardiothorac Vasc Anesth 23:88-107

8. Buckberg G, Hoffman JI, Mahajan A, Saleh S, Coghlan C (2008) Cardiac mechanics revisited: the relationship of cardiac architecture to ventricular function. Circulation 118:2571-2587

9. Lancellotti P, Tribouilloy C, Hagendorff A, Popescu BA, Edvardsen T, Pierard LA et al (2013) Recommendations for the echocardiographic assessment of native valvular regurgitation: an executive summary from the European Association of Cardiovascular Imaging. Eur Heart J Cardiovasc Imag 14:611-644

10. Cheitlin MD, Armstrong WF, Aurigemma GP, Beller GA, Bierman FZ, Davis JL et al (2003) ACC/AHA/ASE 2003 guideline update for the clinical application of echocardiography: summary article: a report of the American College of Cardiology/American Heart Association task force on practice guidelines (ACC/AHA/ ASE committee to update the 1997 guidelines for the clinical application of echocardiography). Circulation 108:1146-1162

11. Dimitriadis Z, Scholtz S, Ensminger S, Wiemer M, Fischbach T, Scholtz W et al (2017) Left ventricular adaptation after TAVI evaluated by conventional and speckle-tracking echocardiography. Int J Cardiol 228:633-637

12. Badano LP, Kolias TJ, Muraru D, Abraham TP, Aurigemma G, Edvardsen $T$ et al (2018) Standardization of left atrial, right ventricular, and right atrial deformation imaging using two-dimensional speckle tracking echocardiography: a consensus document of the EACVI/ASE/Industry Task Force to standardize deformation imaging. Eur Heart J Cardiovasc Imag 19:591-600

13. Haugaa KH, Grenne BL, Eek CH, Ersbøll M, Valeur N, Svendsen JH et al (2013) Strain echocardiography improves risk prediction of ventricular arrhythmias after myocardial infarction. JACC Cardiovasc Imag 6:841-850

14. Witkowski TG, Thomas JD, Debonnaire PJ, Delgado V, Hoke U, Ewe SH et al (2013) Global longitudinal strain predicts left ventricular dysfunction after mitral valve repair. Eur Heart J Cardiovasc Imag 14:69-76

15. Kim HM, Cho GY, Hwang IC, Choi HM, Park JB, Yoon YE et al (2018) Myocardial strain in prediction of outcomes after surgery for severe mitral regurgitation. JACC Cardiovasc Imag 11:1235-1244

16. Haugaa KH, Smedsrud MK, Steen T, Kongsgaard E, Loennechen JP, Skjaerpe T et al (2010) Mechanical dispersion assessed by myocardial strain in patients after myocardial infarction for risk prediction of ventricular arrhythmia. JACC Cardiovasc Imag 3:247-256

17. Haland TF, Almaas VM, Hasselberg NE, Saberniak J, Leren IS, Hopp E et al (2016) Strain echocardiography is related to fibrosis and ventricular arrhythmias in hypertrophic cardiomyopathy. Eur Heart J Cardiovasc Imag 17:613-621

18. Candan O, Hatipoglu Akpinar S, Dogan C, Demirkiran A, Dindar B, Bayram Z et al (2017) Twist deformation for predicting postoperative left ventricular function in patients with mitral regurgitation: a speckle tracking echocardiography study. Echocardiography $34: 422-428$ 
19. Gaasch WH, Shah SP, Labib SB, Meyer TE (2017) Impedance to retrograde and forward flow in chronic mitral regurgitation and the physiology of a double outlet ventricle. Heart 103:581-585

20. Imasaka K, Tomita Y, Tanoue $\mathrm{Y}$, Tominaga R, Tayama E, Onitsuka $\mathrm{H}$ et al (2013) Early mitral valve surgery for chronic severe mitral regurgitation optimizes left ventricular performance and left ventricular mass regression. J Thorac Cardiovasc Surg 146:61-66

21. Unsworth B, Casula RP, Kyriacou AA, Yadav H, Chukwuemeka A, Cherian A et al (2010) The right ventricular annular velocity reduction caused by coronary artery bypass graft surgery occurs at the moment of pericardial incision. Am Heart J 159:314-322

22. Tamborini G, Muratori M, Brusoni D, Celeste F, Maffessanti F, Caiani EG et al (2009) Is right ventricular systolic function reduced after cardiac surgery? A two- and three-dimensional echocardiographic study. Eur J Echocardiogr 10:630-634

23. Zanobini M, Saccocci M, Tamborini G, Veglia F, Di Minno A, Poggio P et al (2017) Postoperative echocardiographic reduction of right ventricular function: is pericardial opening modality the main culprit? BioMed Res Int 2017:4808757
24. Roshanali F, Yousefnia MA, Mandegar MH, Rayatzadeh H, Alinejad S (2008) Decreased right ventricular function after coronary artery bypass grafting. Tex Heart Inst J 35:250-255

25. Katsi V, Raftopoulos L, Aggeli C, Vlasseros I, Felekos I, Tousoulis D et al (2012) Tricuspid regurgitation after successful mitral valve surgery. Interactive CardioVasc Thorac Surg 15:102-108

26. Tamborini G, Fusini L, Muratori M, Gripari P, Ghulam Ali S, Fiorentini $C$ et al (2016) Right heart chamber geometry and tricuspid annulus morphology in patients undergoing mitral valve repair with and without tricuspid valve annuloplasty. Int J Cardiovasc Imag 32:885-894

Publisher's Note Springer Nature remains neutral with regard to jurisdictional claims in published maps and institutional affiliations. 